\title{
A Difference Scheme and Its Error Analysis for a Poisson Equation with Nonlocal Boundary Conditions
}

\author{
Chunsheng Feng $\mathbb{D}^{1},{ }^{1}$ Cunyun Nie, ${ }^{2}$ Haiyuan Yu $\mathbb{D},{ }^{1}$ and Liping Zhou ${ }^{3}$ \\ ${ }^{1}$ Hunan Key Laboratory for Computation and Simulation in Science and Engineering, \\ School of Mathematics and Computational Science, Xiangtan University, Xiangtan 411105, China \\ ${ }^{2}$ School of Mathematics and Physics, Hunan Institution of Engineering, Xiangtan 411105, China \\ ${ }^{3}$ College of Science, Hunan University of Science and Engineering, Yongzhou 425199, China \\ Correspondence should be addressed to Chunsheng Feng; spring@xtu.edu.cn and Haiyuan Yu; 2857643924@qq.com
}

Received 14 July 2020; Revised 12 August 2020; Accepted 17 September 2020; Published 29 September 2020

Academic Editor: Karthikeyan Rajagopal

Copyright (C) 2020 Chunsheng Feng et al. This is an open access article distributed under the Creative Commons Attribution License, which permits unrestricted use, distribution, and reproduction in any medium, provided the original work is properly cited.

\begin{abstract}
The elliptic problem with a nonlocal boundary condition is widely applied in the field of science and engineering, such as the chaotic system. Firstly, we construct one high-accuracy difference scheme for a kind of elliptic problem by tactfully introducing an equivalent relation for one nonlocal condition. Then, we obtain the local truncation error equation by the Taylor formula and, initially, prove that the new scheme can reach the asymptotic optimal error estimate $O\left(h^{2}|\ln h|\right)$ in the maximum norm through ingeniously transforming a two-dimensional problem to a one-dimensional one through bringing in the discrete Fourier transformation. Numerical experiments demonstrate the correctness of theoretical results.
\end{abstract}

\section{Introduction}

Nonlocal boundary value problems have certainly been one of the fastest growing areas in various application fields, such as chaos, chemistry, biology, and physics [1-7]. Some researchers are interested in numerical methods mainly including finite difference methods, finite element methods, finite volume methods, and other methods [8-16]. Many people have paid close attention to the finite difference method for stationary problems, for instance, the Poisson equation [17-22]. Recently, Zhai et al. put forward some compact four-order and six-order difference schemes for a 2-D Poisson equation but lack theoretical analysis [17]. Some people have studied nonlinear elliptic problems with a nonlocal boundary condition. In 2016, Themistoclakis and Vecchio studied a nonlinear boundary value problem involving a nonlocal operator and proposed a classical numerical algorithm to solve the algebraic system by means of some iterative procedures [18]. Cannon developed a numerical method for a homogeneous, nonlinear, nonlocal, elliptic boundary value problem and proved the existence and uniqueness by a continuous compact mapping and the Brouwer fixed point theorem [19]. Pao and Wang concerned with some numerical methods for a fourth-order semilinear elliptic boundary value problem with nonlocal boundary condition. The fourth-order equation was formulated as a coupled system of two second-order equations which were discretized by the finite difference method [20]. Based on fast discrete Sine transform, Wang et al. designed a fast solver to implement a fourth-order compact finite difference scheme for 1-D, 2-D, and 3-D Poisson equations [21]. Islam et al. developed a collocation method based on the Haar wavelet and a meshless method by analyzing for the solution of a two-dimensional Poisson equation with two different types of nonlocal boundary conditions [22].

Other researchers are interested in parabolic problems [23-27]. Ivanauskas et al. discussed the spectrum of a finite difference operator subject to nonlocal Robin-type boundary conditions and analyzed the spectral properties of finite difference schemes for parabolic equations and also discussed alternating direction methods and constructed some weighted splitting finite difference scheme [23-25]. In 2011, 
Ismailov et al. investigated the inverse problem of finding a time-dependent heat source in a parabolic equation with a nonlocal boundary and integral over determination conditions and showed the existence, uniqueness, and continuous dependence upon the data of the solution by using the generalized Fourier method [26, 27]. However, with the scope of the authors' knowledge, there are few literatures that both presented some high-accuracy schemes and showed a theoretical proof for a 2-D elliptic problem with two nonlocal conditions and, furthermore, displayed the corresponding numerical tests. It urges us to go deeply into this problem.

In the present paper, the first novel idea is that we ingeniously construct one high-accuracy difference scheme for a kind of elliptic problem with two nonlocal boundary conditions by introducing an equivalent relations for one nonlocal condition when the solution $u \in C^{4}(\bar{\Omega})$. The local truncation error equation is obtained by the Taylor formula. The second one is that we initially prove that it is convergent with an asymptotic optimal convergent order of two through tactful transforming a two-dimensional problem to a onedimensional one by bringing in the discrete Fourier transformation. Numerical tests confirm the correctness of theoretical results.

The remainder of this paper is organized as follows. In section 2, we display the model problem and its discrete scheme. In section 3, we present error estimate by the discrete Fourier transformation. In section 4, we display numerical experiments to support our conclusions. Finally, we draw some conclusions from this paper.

\section{The Model Problem and the Difference Scheme}

We consider the following second-order elliptic problem with local and nonlocal boundary conditions:

$$
\begin{cases}\Delta u=f(x, y), & (x, y) \in \Omega=(0,1)^{2}, \\ \left.u\right|_{x=0}=\mu_{1}(y), & 0<y<1, \\ \left.u\right|_{x=1}=\mu_{2}(y), & 0<y<1, \\ \left.u\right|_{y=0}-\left.\gamma u\right|_{y=1}=\mu_{3}(y), & 0<x<1, \\ \int_{0}^{1} u \mathrm{~d} y=\mu_{4}(x), & 0<x<1,\end{cases}
$$

where $f(x, y), \mu_{i}(y), i=1,2, \mu_{j}(x), j=3,4$ are some given smooth functions and $\gamma$ is a constant.

To be convenient to discretize the nonlocal boundary, we present an equivalent relation as follows.

Lemma 1. Assume that the solution $u \in C^{2}(\bar{\Omega})$ in Problem (1) and that functions $\mu_{i}(y), i=1,2, \mu_{4}(x)$ satisfy consistent properties as follows:

$$
\begin{aligned}
& \int_{0}^{1} \mu_{1}(y) \mathrm{d} y=\mu_{4}(0), \\
& \int_{0}^{1} \mu_{2}(y) \mathrm{d} y=\mu_{4}(1),
\end{aligned}
$$

and then, the boundary condition $\int_{0}^{1} u d y=\mu_{4}(x)$ is equivalent to the following nonlocal boundary condition:

$$
\left.u_{y}\right|_{y=1}-\left.u_{y}\right|_{y=0}=\int_{0}^{1} f(x, y) \mathrm{d} y-\mu_{4}^{\prime \prime}(x) .
$$

Proof. Integrating two sides of equation (1) about the variable yover the interval $[0,1]$ and noticing that condition $\int_{0}^{1} u \mathrm{~d} y=\mu_{4}(x)$, we have

$$
\mu_{4}^{\prime \prime}(x)+\int_{0}^{1} u_{y y} \mathrm{~d} y=\int_{0}^{1} u_{x x} \mathrm{~d} y+\int_{0}^{1} u_{y y} \mathrm{~d} y=\int_{0}^{1} f(x, y) \mathrm{d} y .
$$

That is,

$$
\left.u_{y}\right|_{y=1}-\left.u_{y}\right|_{y=0}=\int_{0}^{1} f(x, y) \mathrm{d} y-\mu_{4}^{\prime \prime}(x) .
$$

On the other hand, when Condition (3) holds, together with equation (1), we can obtain

$$
\int_{0}^{1} u_{x x} \mathrm{~d} y=\mu_{4}^{\prime \prime}(x)
$$

Integrating twice for two sides of the abovementioned expression about the variable $x$, we have

$$
\int_{0}^{1} u \mathrm{~d} y=\mu_{4}(x)+C_{1} x+C_{2}
$$

where $C_{1}$ and $C_{2}$ are two constants.

From the boundary and consistent conditions $\left.u\right|_{x=0}=\mu_{1}(y), \quad \int_{0}^{1} \mu_{1}(y) \mathrm{d} y=\mu_{4}(0),\left.\quad u\right|_{x=1}=\mu_{2}(y), \quad$ and $\int_{0}^{1} \mu_{1}(y) d y=\mu_{4}(1)$, respectively,

$$
C_{1}=C_{2}=0 \text {. }
$$

Hence,

$$
\int_{0}^{1} u \mathrm{~d} y=\mu_{4}(x)
$$

This completes the proof of this lemma.

In the following, we will present the finite difference scheme for Problem (1) by utilizing Lemma 1.

We take the following partition for Region $\Omega$ along the directions of $x$ and $y$ axes, respectively.

$$
\begin{aligned}
& 0=x_{0}<x_{1}<\cdots<x_{N}=1, \\
& 0=y_{0}<y_{1}<\cdots<y_{N}=1,
\end{aligned}
$$

where $x_{i}=i h, y_{j}=j h, h=(1 / N)$, and $N$ is the corresponding partition number.

Equation (1) and two local boundary conditions can be discretized as follows: 


$$
\begin{cases}\frac{U_{i-1, j}-2 U_{i, j}+U_{i+1, j}}{h^{2}}+\frac{U_{i, j-1}-2 U_{i, j}+U_{i, j+1}}{h^{2}}=f_{i, j}, & i, j=1,2, \ldots, N-1, \\ U_{0, j}=\left(\mu_{1}\right)_{j}, U_{N, j}=\left(\mu_{2}\right)_{j}, & j=1,2, \ldots, N-1,\end{cases}
$$

where $u_{i, j}$ and $U_{i, j}$ are the exact and approximate solutions of Problem (1) at Point $\left(x_{i}, y_{j}\right)$, respectively.
From Lemma 1, two nonlocal boundary conditions can be discretized as follows:

$$
\left\{\begin{array}{l}
U_{i, 0}=\gamma U_{i, N}+\left(\mu_{3}\right)_{i} \\
\frac{1}{h}\left(\frac{3}{2} U_{i, N}-2 U_{i, N-1}+\frac{1}{2} U_{i, N-2}\right)-\frac{1}{h}\left(-\frac{3}{2} U_{i, 0}+2 U_{i, 1}-\frac{1}{2} U_{i, 2}\right)=\varphi_{i} \\
i=1,2, \ldots, N-1
\end{array}\right.
$$

where $\varphi_{i}=\int_{0}^{1} f\left(x_{i}, y\right) \mathrm{d} y-\mu_{4}^{\prime \prime}\left(x_{i}\right)$.

\section{Error Estimate}

To be convenient, we introduce the denotation $A \leqslant B$, which means that there exists some constant $C_{1}>0$ such that $A \leq C_{1} B$.
Assume that the solution $u \in C^{4}(\bar{\Omega})$ in Problem (1), from (11) and (12), and combining with equation (1), we have

$$
\begin{aligned}
& \begin{cases}\frac{e_{i-1, j}-2 e_{i, j}+e_{i+1, j}}{h^{2}}+\frac{e_{i, j-1}-2 e_{i, j}+e_{i, j+1}}{h^{2}}=h^{2} \alpha_{i, j}, & i, j=1,2, \ldots, N-1, \\
e_{0, j}=e_{N, j}=0, & j=1,2, \ldots, N-1,\end{cases} \\
& \left\{\begin{array}{l}
e_{i, 0}=\gamma e_{i, N}, \\
\frac{1}{h}\left(\frac{3}{2} e_{i, N}-2 e_{i, N-1}+\frac{1}{2} e_{i, N-2}\right)-\frac{1}{h}\left(-\frac{3}{2} e_{i, 0}+2 e_{i, 1}-\frac{1}{2} e_{i, 2}\right)=h^{2} \beta_{i}, \\
i=1,2, \ldots, N-1,
\end{array}\right.
\end{aligned}
$$

where $e_{i, j}=U_{i, j}-u_{i, j}(i, j=0,1, \ldots, N)$ is the error of the finite difference solution at Point $\left(x_{i}, y_{j}\right)$ and $\alpha_{i, j}, \beta_{i}$ are coefficients of the corresponding local truncation errors, respectively, and they satisfy

$$
\begin{gathered}
\left|\alpha_{i, j}\right| \lesssim M_{4}, \\
\left|\beta_{i}\right| \lesssim M,
\end{gathered}
$$

and $M_{k}=|u|_{k, \infty}, M=\max \left\{M_{3}, M_{4}\right\}$.

Firstly, we will introduce the following discrete Fourier transformation:

$$
e_{i, j}=\sqrt{2 h} \sum_{k=1}^{N-1} \widehat{e}_{k, j} \sin k \pi x_{i}, \quad i, j=1,2, \ldots, N-1 .
$$

Similar transformations for $\alpha_{i, j}, \beta_{i}$, respectively, are as follows:

$$
\begin{cases}\alpha_{i, j}=\sqrt{2 h} \sum_{k=1}^{N-1} \widehat{\alpha}_{k, j} \sin k \pi x_{i}, & i, j=1,2, \ldots, N-1, \\ \beta_{i}=\sqrt{2 h} \sum_{k=1}^{N-1} \widehat{\beta}_{k} \sin k \pi x_{i}, & i=1,2, \ldots, N-1 .\end{cases}
$$

Due to the fact that $\sqrt{2 h}\left(\sin k \pi x_{i}\right)_{(N-1) \times(N-1)}$ is an orthogonal matrix, the following inverse transformation formulas hold: 


$$
\begin{cases}\widehat{\alpha}_{k, j}=\sqrt{2 h} \sum_{i=1}^{N-1} \alpha_{i, j} \sin i \pi x_{k}, & k, j=1,2, \ldots, N-1, \\ \widehat{\beta}_{k}=\sqrt{2 h} \sum_{i=1}^{N-1} \beta_{i} \sin i \pi x_{k}, & k=1,2, \ldots, N-1 .\end{cases}
$$

From (15), we have

$$
\begin{gathered}
\left|\widehat{\beta}_{k}\right| \lesssim h^{-(1 / 2)}, \\
\left|\widehat{\alpha}_{k, j}\right| \lesssim h^{-(1 / 2)} .
\end{gathered}
$$

Taking the discrete Fourier transformation for equations (13) and (14), respectively, for the variable $i$, we have

$$
\left\{\begin{array}{l}
\widehat{e}_{k, j-1}-\omega_{k} \widehat{e}_{k, j}+\widehat{e}_{k, j+1}=h^{4} \widehat{\alpha}_{k, j}, \quad j=1,2, \ldots, N-1, \\
\widehat{e}_{k, 0}=\gamma \widehat{e}_{k, N}, \\
\frac{3}{2} \widehat{e}_{k, N}-2 \widehat{e}_{k, N-1}+\frac{1}{2} \widehat{e}_{k, N-2}+\frac{3}{2} \widehat{e}_{k, 0}-2 \widehat{e}_{k, 1}+\frac{1}{2} \widehat{e}_{k, 2}=h^{3} \widehat{\beta}_{k},
\end{array}\right.
$$

where

$$
\begin{aligned}
& \omega_{k}=2+4 \sin ^{2} \theta_{k}, \\
& \theta_{k}=\frac{k \pi h}{2} .
\end{aligned}
$$

Let

$$
\varepsilon_{k, j}=\widehat{e}_{k, j}+h^{4} p_{k, j}
$$

where $p_{k, j}$ satisfies

$$
\left\{\begin{array}{l}
-p_{k, j-1}+\omega_{k} p_{k, j}-p_{k, j+1}=\widehat{\alpha}_{k, j}, \quad j=1,2, \ldots, N-1, \\
p_{k, 0}=p_{k, N}=0 .
\end{array}\right.
$$

From (21) and (24), one can see that

$$
\left\{\begin{array}{l}
\varepsilon_{k, j-1}-\omega_{k} \varepsilon_{k, j}+\varepsilon_{k, j+1}=0, \quad j=1,2, \ldots, N-1, \\
\varepsilon_{k, 0}=\gamma \varepsilon_{k, N}, \\
\frac{3}{2} \varepsilon_{k, N}-2 \varepsilon_{k, N-1}+\frac{1}{2} \varepsilon_{k, N-2}+\frac{3}{2} \varepsilon_{k, 0}-2 \varepsilon_{k, 1}+\frac{1}{2} \varepsilon_{k, 2}=h^{3} \widetilde{\beta}_{k},
\end{array}\right.
$$

where

$$
\tilde{\beta}_{k}=\widehat{\beta}_{k}+h\left(\frac{3}{2} p_{k, N}-2 p_{k, N-1}+\frac{1}{2} p_{k, N-2}+\frac{3}{2} p_{k, 0}-2 p_{k, 1}+\frac{1}{2} p_{k, 2}\right) .
$$

Let

$$
\left\|\widehat{\alpha}_{k}\right\|=\max _{j=1, \ldots, N-1}\left|\widehat{\alpha}_{k, j}\right| \cdot
$$

Now, we can obtain the following estimates.

Lemma 2. Suppose that $p_{k, j}$ satisfies (24). Then, we have

$$
\begin{array}{r}
\max _{j=1, \ldots, N-1}\left|p_{k, j}\right| \lesssim \frac{h^{-2}}{k^{2}}\left\|\widehat{\alpha}_{k}\right\| . \\
\max _{j=0, \ldots, N-1}\left|p_{k, j+1}-p_{k, j}\right| \lesssim h^{-1}\left\|\widehat{\alpha}_{k}\right\| .
\end{array}
$$

Proof. Let $\left|p_{k, \ell}\right|=\max _{j=1, \ldots, N-1}\left|p_{k, j}\right|$. Then, from (22) and (24), we have

$$
\left|\widehat{\alpha}_{k, \ell}\right|=\left|-p_{k, \ell-1}+\omega_{k} p_{k, \ell}-p_{k, \ell+1}\right| \geq\left(\omega_{k}-2\right)\left|p_{k, \ell}\right|=4 \sin ^{2} \theta_{k}\left|p_{k, \ell}\right| \text {. }
$$

Recalling that $\theta_{k}=(k \pi h / 2), \quad h=(1 / N) \quad$ and $1 \leq k \leq N-1$, we get $\theta_{k} \in(0,(\pi / 2))$.

Moreover,

$$
\sin \theta_{k} \geq \frac{2}{\pi} \theta_{k}=\mathrm{kh}
$$

Therefore, one can easily infer (28).

Let $\delta_{k, i}=\widehat{\alpha}_{k, i}-4 \sin ^{2} \theta_{k} p_{k, i}, \quad i=1,2, \ldots, N-1$. From (30), we have

$$
\left|\delta_{k, i}\right| \leq 2\left\|\widehat{\alpha}_{k}\right\|
$$

From (24), we get

$$
-p_{k, i-1}+2 p_{k, i}-p_{k, i+1}=\delta_{k, i}
$$

Then, summing the abovementioned equation overi from 1 to $j(1 \leq J \leq N-1)$, we obtain

$$
p_{k, j+1}-p_{k, j}=p_{k, 1}-p_{k, 0}-\sum_{i=1}^{j} \delta_{k, i} .
$$

Furthermore, summing (34) over $j$ from 1 to $N-1$ and noticing $p_{k, N}=p_{k, N}=0$, we get

$$
-\left(p_{k, 1}-p_{k, 0}\right)=(N-1)\left(p_{k, 1}-p_{k, 0}\right)-\sum_{j=1}^{N-1} \sum_{i=1}^{j} \delta_{k, i} .
$$

From (32) and the abovementioned equation, one can obtain

$$
\left|p_{k, 1}-p_{k, 0}\right| \leq h^{-1}\left\|\widehat{\alpha}_{k}\right\| .
$$

Therefore, using (32) again together with (34), 29) holds, which completes the proof.

Theorem 1. Assume that $u \in C^{4}(\bar{\Omega})$ and $U_{i, j}$ are the exact and finite difference solutions for Problem (1); then, as $\gamma \neq-1$, for $i, j=1,2, \ldots, N-1$, we have

$$
U_{i, j}=u_{i, j}+O\left(h^{2}|\ln h|\right) \text {. }
$$




$$
\begin{aligned}
& \lambda_{k}=\left(\sqrt{1+\sin ^{2} \theta_{k}}+\sin \theta_{k}\right)^{2}, \\
& \theta_{k}=\frac{k \pi h}{2},
\end{aligned}
$$

which satisfy

$$
\begin{gathered}
\lambda_{k}+\lambda_{k}^{-1}=\omega_{k}, \\
\sqrt{\lambda_{k}}-\frac{1}{\sqrt{\lambda_{k}}}=2 \sin \theta_{k} .
\end{gathered}
$$

From the former two expressions of (25), we can derive that there exists $C_{k}$ such that

$$
\varepsilon_{k, j}=C_{k}\left[\left(\lambda_{k}^{j}-\lambda_{k}^{-j}\right)+\gamma\left(\lambda_{k}^{N-j}-\lambda_{k}^{-(N-j)}\right)\right] .
$$

In fact, we take the value of $j$ as $N, N-1, N-2,0,1,2$ in (40), respectively, and substitute them into the third expression in (25). Then, we obtain

$$
C_{k}=\frac{h^{3} \widetilde{\beta}_{k}}{\xi_{k}(1+\gamma)}
$$

where

$$
\begin{aligned}
\xi_{k}= & \frac{3}{2}\left(\lambda_{k}^{N}-\lambda_{k}^{-N}\right)-2\left(\lambda_{k}^{N-1}-\lambda_{k}^{1-N}\right)+\frac{1}{2}\left(\lambda_{k}^{N-2}-\lambda_{k}^{2-N}\right) \\
& -2\left(\lambda_{k}-\lambda_{k}^{-1}\right)+\frac{1}{2}\left(\lambda_{k}^{2}-\lambda_{k}^{-2}\right) .
\end{aligned}
$$

From (39) and observing that $\theta_{k} \in(0,(\pi / 2))$ and $\lambda_{k}>1$, we have

$$
\begin{aligned}
\xi_{k}= & \left(\sqrt{\lambda_{k}}-\frac{1}{\sqrt{\lambda_{k}}}\right)\left(\lambda_{k}^{(N / 2)}-\lambda_{k}^{-(N / 2)}\right)\left[\frac{3}{2}\left(\lambda_{k}^{(N-1 / 2)}-\lambda_{k}^{(1-N / 2)}\right)\right. \\
& \left.-\frac{1}{2}\left(\lambda_{k}^{(N-3 / 2)}-\lambda_{k}^{(3-N / 2)}\right)\right] \\
\geq & 2 \sin \theta_{k}\left(\lambda_{k}^{(N / 2)}-\lambda_{k}^{-(N / 2)}\right)\left(\lambda_{k}^{(N-1 / 2)}-\lambda_{k}^{(1-N / 2)}\right) \\
\geq & 2 \operatorname{kh}\left(\lambda_{k}^{(N / 2)}-\lambda_{k}^{-(N / 2)}\right)\left(\lambda_{k}^{(N-1 / 2)}-\lambda_{k}^{(1-N / 2)}\right) .
\end{aligned}
$$

On the other hand, from (26), (19), (22), and Lemma 2, we have

$$
\left|\widetilde{\beta}_{k}\right| \lesssim h^{-(1 / 2)}
$$

Synthesizing the estimates on $\xi_{k}$ and $\widetilde{\beta}_{k}$ : (43) and (44), together with (41), then we have

$$
\left|C_{k}\right| \lesssim \frac{h^{(3 / 2)}}{k}\left(\lambda_{k}^{(N / 2)}-\lambda_{k}^{-(N / 2)}\right)^{-1}\left(\lambda_{k}^{(N-1 / 2)}-\lambda_{k}^{(1-N / 2)}\right)^{-1} .
$$

Furthermore, from (40),

$$
\begin{aligned}
\left|\varepsilon_{k, j}\right| & \lesssim\left|C_{k}\right|\left(\lambda_{k}^{N-1}-\lambda_{k}^{1-N}\right) \\
& \lesssim \frac{h^{(3 / 2)}}{k} \frac{\lambda_{k}^{((N-1) / 2)}+\lambda_{k}^{((1-N) / 2)}}{\lambda_{k}^{(N / 2)}-\lambda_{k}^{-(N / 2)}} \\
& \lesssim \frac{h^{(3 / 2)}}{k} \frac{\lambda_{k}^{(N / 2)}+\lambda_{k}^{-(N / 2)}}{\lambda_{k}^{(N / 2)}-\lambda_{k}^{-(N / 2)}} .
\end{aligned}
$$

Due to the fact that

$$
\begin{aligned}
\lambda_{k}^{(N / 2)} & =\left(\sqrt{1+\sin ^{2} \theta_{k}}+\sin \theta_{k}\right)^{N} \\
& \geq\left(1+\sin \frac{k \pi}{2 N}\right)^{N} \geq\left(1+\frac{k}{N}\right)^{N} \geq\left(1+\frac{1}{N}\right)^{N} \geq 2,
\end{aligned}
$$

we have

$$
\left|\varepsilon_{k, j}\right| \lesssim \frac{h^{(3 / 2)}}{k} .
$$

From Lemma 2 and (20),

$$
\left|\widehat{e}_{k, j}\right|=\left|\varepsilon_{k, j}-h^{4} p_{k, j}\right| \leq\left|\varepsilon_{k, j}\right|+h^{4}\left|p_{k, j}\right| \lesssim \frac{h^{(3 / 2)}}{k}+\frac{h^{2}}{k^{2}}\left|\widehat{\alpha}_{k}\right| \lesssim \frac{h^{(3 / 2)}}{k} \text {. }
$$

Together with the fact that

$$
e_{i, j}=U_{i, j}-u_{i, j}=\sqrt{2 h} \sum_{k=1}^{N-1} \widehat{e}_{k, j} \sin k \pi x_{i},
$$

one can obtain (37). This completes the proof of the theorem.

\section{Numerical Experiments}

In this section, we carry on some numerical experiments for Problem (1).

Example 1. In Problem (1), we take $\mu_{k}(y)=0, k=1,2$, $\mu_{3}(x)=\sin \pi x \mu_{4}(x)=(e-1) \sin \pi x, \gamma=0$, and the exact solution $u(x, y)=e^{y} \sin \pi x$. One can easily see that $f(x, y)=\left(1-\pi^{2}\right) e^{y} \sin \pi x$.

In this experiment, we take the uniform partition for Region $\Omega$ and the step size $h=\left(1 / 2^{k}\right)$, utilize Scheme (11) and (12), and employ the PCG method to solve the corresponding discrete system. Numerical results are shown as Tables 1 and 2, where the norms $\left\|v_{m}\right\|(m=2, \infty)$ are defined as $\left\|v_{2}\right\|=(1 / N)\left(\sum_{i=1}^{N} \sum_{j=1}^{N}\left|v_{i, j}\right|^{2}\right)^{(1 / 2)},\left|v_{\infty}\right|=\max _{1 \leq i, j \leq N}\left|v_{i, j}\right|$, respectively, and $\eta$ is the ratio of the errors between the approximate and exact solutions for step sizes $h$ and $(h / 2)$. In order to display the pointwise error, we show the corresponding errors for four typical points in Table 2. From the results, one can see that the convergent order is two, which validates the correctness of theoretical results. 
TABLE 1: The errors for finite difference solutions in two norms.

\begin{tabular}{ccccc}
\hline$k$ & $\|u-U\|_{2}$ & $\eta$ & $\|u-U\|_{\infty}$ & $\eta$ \\
\hline 4 & $4.632 E-03$ & & $1.198 E-02$ & \\
5 & $1.166 E-03$ & 3.97 & $3.004 E-03$ & \\
6 & $2.924 E-04$ & 3.99 & $7.520 E-04$ & 3.99 \\
7 & $7.325 E-05$ & 3.99 & $1.882 E-04$ & 4.00 \\
\hline
\end{tabular}

TABle 2: The errors for finite difference solutions in the pointwise sense.

\begin{tabular}{|c|c|c|c|c|c|c|c|c|}
\hline$(x, y) k$ & $(0.25,0.25)$ & $\eta$ & $(0.25,0.5)$ & $\eta$ & $(0.5,0.25)$ & $\eta$ & $(0.5,0.5)$ & $\eta$ \\
\hline 4 & $2.240 E-03$ & & $4.011 E-03$ & & $3.168 E-03$ & & $5.672 E-03$ & \\
\hline 5 & $5.601 E-04$ & 4.00 & $1.003 E-03$ & 4.00 & $7.921 E-04$ & 4.00 & $1.419 E-03$ & 4.00 \\
\hline 6 & $1.401 E-04$ & 4.00 & $2.509 E-04$ & 4.00 & $1.981 E-04$ & 4.00 & $3.548 E-04$ & 4.00 \\
\hline 7 & $3.502 E-05$ & 4.00 & $6.274 E-05$ & 4.00 & $4.953 E-05$ & 4.00 & $8.872 E-05$ & 4.00 \\
\hline
\end{tabular}

TABLE 3: The errors for finite difference solutions in two norms.

\begin{tabular}{ccccc}
\hline$k$ & $\|u-U\|_{2}$ & $\eta$ & $\|u-U\|_{\infty}$ & $\eta$ \\
\hline 4 & $1.056 E-03$ & & $1.539 E-03$ & \\
5 & $2.680 E-04$ & 3.94 & $3.848 E-04$ & \\
6 & $6.752 E-05$ & 3.97 & $9.623 E-05$ & 4.00 \\
7 & $1.695 E-05$ & 3.98 & $2.406 E-05$ & 4.00 \\
\hline
\end{tabular}

TABle 4: The errors for finite difference solutions in the pointwise sense.

\begin{tabular}{|c|c|c|c|c|c|c|c|c|}
\hline$(x, y) k$ & $(0.25,0.25)$ & $\eta$ & $(0.25,0.5)$ & $\eta$ & $(0.5,0.25)$ & $\eta$ & $(0.5,0.5)$ & $\eta$ \\
\hline 4 & $9.571 E-04$ & & $9.827 E-04$ & & $1.536 E-05$ & & $1.474 E-03$ & \\
\hline 5 & $2.394 E-04$ & 4.00 & $2.458 E-04$ & 4.00 & $3.591 E-04$ & 4.00 & $3.685 E-04$ & 4.00 \\
\hline 6 & $5.984 E-05$ & 4.00 & $6.144 E-05$ & 4.00 & $8.978 E-05$ & 4.00 & $9.214 E-05$ & 4.00 \\
\hline 7 & $1.496 E-05$ & 4.00 & $1.536 E-05$ & 4.00 & $2.244 E-05$ & 4.00 & $2.304 E-05$ & 4.00 \\
\hline
\end{tabular}

Example 2. In Problem (1), we take $\mu_{1}(y)=y^{2}$, $\mu_{2}(y)=e(1+y)^{2}, \quad \mu_{3}(x)=-e^{x}(2 x+1), \quad \mu_{4}(x)=e^{x}\left(x^{2}+\right.$ $x+(1 / 3)), \gamma=1$, and the exact solution $u=e^{x}(x+y)^{2}$. One can easily obtain $f(x, y)=e^{x}(x+y)^{2}+4 e^{x}(x+y+1)$.

We take the same methods as in Example 1 and get numerical results shown as Tables 3 and 4. From the results, one can see that the convergent order is two, which also confirms the correctness of theoretical results.

\section{Summary and Conclusions}

In this paper, firstly, we construct one high-accuracy difference scheme for a kind of elliptic problem with two nonlocal boundary conditions by introducing an equivalent expression for one nonlocal condition. Secondly, we, initially, prove that it is convergent with a saturated order through ingeniously transforming a two-dimensional problem to a one-dimensional one by bringing in the discrete Fourier transformation. Finally, we carry out some numerical tests to verify the correctness of theoretical results.

\section{Data Availability}

All data generated or analyzed during this study are included in this article.

\section{Conflicts of Interest}

The authors declare that they have no conflicts of interest.

\section{Acknowledgments}

This work was partially supported by National Natural Science Foundation of China (Grant no. 11971414), Hunan Province Science and Technology Innovation Plan Project (Grant no. 2018XK2304), and Youth Project of Hunan Provincial Education Department (Grant nos. 18B518 and 18B082).

\section{References}

[1] J. Zhou and D. Yang, "Legendre-Galerkin spectral methods for optimal control problems with integral constraint for state 
in one dimension," Computational Optimization and Applications, vol. 61, no. 1, pp. 135-158, 2015.

[2] H. Niu, D. Yang, and J. Zhou, "Numerical analysis of an optimal control problem governed by the stationary Navierstokes equations with global velocityconstrained," Communications in Computational Physics, vol. 24, no. 5, pp. 14771502, 2018.

[3] H. Chen, Y. Liu, C. Feng, A. Liu, and X. Huang, "Dynamics at infinity and existence of singularly degenerate heteroclinic cycles in Maxwell-Bloch system," Journal of Computational and Nonlinear Dynamics, vol. 15, no. 10, p. 8, Article ID 101007, 2020.

[4] B. Chen, Y. Liu, Z. Wei, and C. Feng, "New insights into a chaotic system with only a Lyapunov stable equilibrium," Mathematical Methods in the Applied Sciences, vol. 43, no. 15, pp. 9262-9279, 2020.

[5] C. Feng, Q. Huang, and Y. Liu, "Jacobi analysis for an unusual 3D autonomous system," International Journal of Geometric Methods in Modern Physics, vol. 17, no. 4, p. 20, Article ID 2050062, 2020.

[6] C. Feng, L. Li, Y. Liu, and Z. Wei, "Global dynamics of the chaotic disk dynamo system driven by noise," Complexity, vol. 2020, no. 33, 9 pages, Article ID 8375324, 2020.

[7] Y. Liu, Z. Liu, and D. Motreanu, "Existence and approximated results of solutions for a class of nonlocal elliptic variationalhemivariational inequalitie," Mathematical Methods in the Applied Sciences, pp. 1-14, 2020.

[8] R. S. Hirsh, "Higher order accurate difference solutions of fluid mechanics problems by a compact differencing technique," Journal of Computational Physics, vol. 19, no. 1, pp. 90-109, 1975.

[9] C. V. Pao, "Numerical solutions of reaction-diffusion equations with nonlocal boundary conditions," Journal of Computational and Applied Mathematics, vol. 136, no. 1-2, pp. 227-243, 2001.

[10] R. Ma, "A survey on nonlocal boundary value problems," Applied Mathematics E-Notes, vol. 7, pp. 257-279, 2007.

[11] G. K. Berikelashvili and N. Khomeriki, "On a numerical solution of one nonlocal boundary-value problem with mixed Dirichlet-Neumann conditions," Lithuanian Mathematical Journal, vol. 53, no. 4, pp. 367-380, 2013.

[12] C. Nie and H. Yu, "Some error estimates on the finite element approximation for two-dimensional elliptic problem with nonlocal boundary," Applied Numerical Mathematics, vol. 68, pp. 31-38, 2013.

[13] Y. Zhang, "Optimal error estimates of compact finite difference discretizations for the schrödinger-Poisson system," Communications in Computational Physics, vol. 13, no. 5, pp. 1357-1388, 2013.

[14] C. Nie, S. Shu, H. Yu, and Q. An, "A high order composite scheme for the second order elliptic problem with nonlocal boundary and its fast algorithm," Applied Mathematics and Computation, vol. 227, pp. 212-221, 2014.

[15] T. Wang, "Optimal point-wise error estimate of a compact difference scheme for the coupled Gross-Pitaevskii equations in one dimension," Journal of Scientific Computing, vol. 59, no. 1, pp. 158-186, 2014.

[16] J. Zhou, Z. Jiang, H. Xie, and H. Niu, “The error estimates of spectral methods for 1-dimension singularly perturbed problem," Applied Mathematics Letters, vol. 100, Article ID 106001, 2020.

[17] S. Zhai, X. Feng, and Y. He, "A new method to deduce highorder compact difference schemes for two-dimensional
Poisson equation," Applied Mathematics and Computation, vol. 230, pp. 9-26, 2014.

[18] W. Themistoclakis and A. Vecchio, "On the numerical solution of a nonlocal boundary value problem," Journal of Computational and Applied Mathematics, vol. 292, pp. 720731, 2016.

[19] J. R. Cannon and D. J. Galiffa, "On a numerical method for a homogeneous, nonlinear, nonlocal, elliptic boundary value problem," Nonlinear Analysis: Theory, Methods \& Applications, vol. 74, no. 5, pp. 1702-1713, 2011.

[20] C. V. Pao and Y.-M. Wang, "Numerical methods for fourthorder elliptic equations with nonlocal boundary conditions," Journal of Computational and Applied Mathematics, vol. 292, pp. 447-468, 2016.

[21] H. Wang, Y. Zhang, X. Ma, J. Qiu, and Y. Liang, "An efficient implementation of fourth-order compact finite difference scheme for Poisson equation with Dirichlet boundary conditions," Computers \& Mathematics with Applications, vol. 71, no. 9, pp. 1843-1860, 2016.

[22] S.-U. Islam, A. Imran, and M. Ahmad, "Numerical solution of two-dimensional elliptic PDEs with nonlocal boundary conditions," Computers and Mathematics with Applications, vol. 69, no. 3, pp. 180-205, 2015.

[23] F. Ivanauskas, T. Meškauskas, and M. Sapagovas, "Stability of difference schemes for two-dimensional parabolic equations with non-local boundary conditions," Applied Mathematics and Computation, vol. 215, no. 7, pp. 2716-2732, 2009.

[24] M. Sapagovas, T. Meškauskas, and F. Ivanauskas, "Numerical spectral analysis of a difference operator with non-local boundary conditions," Applied Mathematics and Computation, vol. 218, no. 14, pp. 7515-7527, 2012.

[25] S. Sajavĩcius, "Stability of the weighted splitting finite-difference scheme for a two-dimensional parabolic equation with two nonlocal integral conditions," Computers and Mathematics with Applications, vol. 64, no. 11, pp. 3485-3499, 2012.

[26] M. I. Ismailov, F. Kanca, and D. Lesnic, "Determination of a time-dependent heat source under nonlocal boundary and integral overdetermination conditions," Applied Mathematics and Computation, vol. 218, no. 8, pp. 4138-4146, 2011.

[27] A. Hazanee and D. Lesnic, "Determination of a time-dependent coefficient in the bioheat equation," International Journal of Mechanical Sciences, vol. 88, pp. 259-266, 2014. 